\title{
Isospin asymmetric matter in a nonlocal chiral quark model
}

\author{
J. P. Carlomagno, ${ }^{1, *}$ D. Gómez Dumm $\odot,{ }^{1}$ and N. N. Scoccola $\oplus^{2,3}$ \\ ${ }^{1}$ IFLP, CONICET-Departamento de Física, Facultad de Ciencias Exactas, \\ Universidad Nacional de La Plata, C.C. 67, 1900 La Plata, Argentina \\ ${ }^{2}$ CONICET, Rivadavia 1917, 1033 Buenos Aires, Argentina \\ ${ }^{3}$ Physics Department, Comisión Nacional de Energía Atómica, Avenida del Libertador 8250, \\ 1429 Buenos Aires, Argentina
}

(Received 9 July 2021; accepted 13 September 2021; published 15 October 2021)

\begin{abstract}
We analyze the features of strongly interacting matter in the presence of nonzero isospin chemical potential $\mu_{I}$, within a nonlocal two-flavor Polyakov-loop extended Nambu-Jona-Lasinio (PNJL) model. For a system at finite temperature $T$, we describe the behavior of various thermodynamic quantities and study the phase diagram in the $\mu_{I}-T$ plane. In particular, it is found that for values of $\mu_{I}$ larger than the pion mass and temperatures lower than a critical value of about $170 \mathrm{MeV}$ the system lies in an isospin symmetry broken phase signalled by the presence of a nonzero pion condensate. Our results for the phase diagram are found to be in better agreement with those arising from lattice QCD calculations, as compared to the predictions from other theoretical approaches like the local PNJL model.
\end{abstract}

DOI: 10.1103/PhysRevD.104.074018

\section{INTRODUCTION}

The phase diagram of strongly interacting matter at finite temperature and chemical potential has been extensively studied along the past decades. In general, in the region of very high temperatures and low densities it is known that quantum chromodynamics $(\mathrm{QCD})$ predicts the formation of a quark-gluon plasma (QGP) [1]. Under these extreme conditions quarks and gluons are expected to be weakly coupled, and the phase diagram can be explored by means of firstprinciple perturbative calculations based on expansions in powers of the QCD coupling constant. On the other hand, at vanishing baryon chemical potential $\mu_{B}$ one also expects a transition from the hadronic phase to a QGP phase. Lattice QCD (LQCD) calculations indicate that this passage should occur in the form of a smooth crossover, at a pseudocritical temperature $T_{\mathrm{pc}} \sim 150-170 \mathrm{MeV}$. Unfortunately, LQCD calculations at finite baryon chemical potential $\mu_{B}$ are not accessible by Monte Carlo simulations, due to the presence of a complex fermion determinant in the corresponding partition function (the so-called sign problem). In this region, which is not accessible either through lattice techniques or first principles, most of the present theoretical knowledge on

*carlomagno@fisica.unlp.edu.ar

Published by the American Physical Society under the terms of the Creative Commons Attribution 4.0 International license. Further distribution of this work must maintain attribution to the author(s) and the published article's title, journal citation, and DOI. Funded by SCOAP ${ }^{3}$. the QCD phase diagram is obtained from the study of effective models for strong interactions.

At sufficiently high densities and low temperatures, one expects to find a "color-flavor locked" phase [2], in which quarks are bound in color superconducting states analogous to the Cooper pairs formed by electrons in an ordinary superconductor. In the region of moderate values of $\mu_{B}$ the description of the QCD phase diagram is presently an open issue, and various possible scenarios have been discussed in the past few decades. For low temperatures it has been noted that the chiral symmetry restoration might occur within a range of chemical potentials in which quarks are still confined, giving rise to a so-called "quarkyonic" phase. The presence of this phase has been conjectured for large$N_{C}$ QCD [3] and has been studied in the context of effective models for strong interactions $[4,5]$ (astrophysical consequences of the quarkyonic matter state can be found e.g., in Refs. [6,7]). In fact, it has been argued [8,9] that at zero temperature there could be a continuous connection between hadronic and quark phases of dense matter, instead of the usual picture in which a first-order transition exists between both phases at a given critical chemical potential $\mu_{c}$. Support in favor of this continuity scenario has also been found on the basis of a Ginzburg-Landau analysis, which leads to a first-order transition line that ends at a critical endpoint before reaching the $\mu_{B}$ axis in the $\mu_{B}-T$ phase diagram [10]. In addition, it has been claimed that for large chemical potentials a transition to nonuniform phases might be favored, leading to the presence of a Lifshitz point where two homogeneous and one inhomogeneous phases meet (see e.g., Refs. [11-14]). 
Taking into account the difficulties of applying LQCD techniques to study the region of moderate and large chemical potentials, the role played by effective models in the understanding of the QCD phase diagram is particularly relevant, and it is important to test their reliability. This can be done by comparing the corresponding predictions with those obtained from first-principle calculations, in situations where the latter are available. One obvious possibility is to consider the above mentioned case of strong-interaction matter at finite temperature and vanishing chemical potential, in which LCQD calculations do not suffer from the sign problem. Another interesting situation is the one in which the baryon chemical potential $\mu_{B}$ is zero, but one has a nonzero isospin chemical potential $\mu_{I}$. In this case (both at zero and finite temperature) LQCD simulations are feasible, since the functional determinant turns out to be real [15]. Moreover, the study of the phase diagram at finite $\mu_{I}$ becomes interesting in view of the orbifold equivalence between large- $N_{C} \mathrm{QCD}$ and "parent" $\mathrm{SO}\left(2 N_{C}\right)$ and $\operatorname{Sp}\left(2 N_{C}\right)$ Yang-Mills theories, at finite $\mu_{B}$ $[16,17]$. As discussed in Ref. [18], on this basis one also expects an connection between phase diagrams for QCD at finite $\mu_{B}$ and at $\mu_{B}=0$ and finite $\mu_{I}$. This relation is expected to hold even beyond the large $N_{c}$ limit, as long as baryon number symmetry is kept unbroken.

Following the early work in Refs. [19,20], several groups have performed LQCD calculations at $\mu_{I} \neq 0$ using different techniques, see e.g., Refs. [21-26]. One important feature confirmed by these calculations is that at $\mu_{I} \gtrsim m_{\pi}$ one finds the onset of a Bose-Einstein pion condensation phase, as previously conjectured in Ref. [27]. For a recent review on meson condensation triggered by a large isospin imbalance see Ref. [28], where references to various theoretical approaches for the analysis of associated phase transitions can be found. In this work we consider the properties of quark matter at finite isospin chemical potential using a particular class of effective theories, viz. the nonlocal Polyakov-loop extended Nambu-JonaLasinio (nIPNJL) models [29-34]. In the nlPNJL approach the quarks move in a background color field and interact through covariant nonlocal chirally symmetric four-point couplings, which are separable in momentum space. At vanishing $\mu_{B}$ and finite temperature these models provide a plausible description of chiral restoration and deconfinement transitions, in good agreement with LQCD results [35]. In general, it can be considered that they represent an improvement over the local Polyakov-loop extended Nambu-Jona-Lasinio (PNJL) model [36-42]. In fact, nonlocal interactions arise naturally in the context of several successful approaches to low-energy quark dynamics, and lead to a momentum dependence in quark propagators that can be made consistent [43] with lattice results. Moreover, it can be seen that nonlocal extensions of the NJL model do not show some of the known inconveniences that are present in the local theory. Well-behaved nonlocal form factors can regularize the loop integrals in such a way that anomalies are preserved [44] and charges are properly quantized. In addition, one can avoid the introduction of various sharp cutoffs to deal with higherorder loop integrals [45], improving in this way the predictive power of the models.

Within the aforementioned framework, the aim of the present work is to provide a comparison, both at zero and finite temperature, between the results obtained within the nIPNJL model and those arising from other theoretical approaches. In particular, we consider the results from the local NJL model [46,47], its PNJL extension [48,49], chiral perturbation theory (ChPT) [50-52], and recent LQCD calculations $[25,26]$. As shown below, our predictions for the border of the pion condensation phase are in good agreement with available results from lattice QCD, whereas they differ significantly from the predictions obtained in the framework of ChPT and from those found within the local PNJL model.

This article is organized as follows. In Sec. II we present the general formalism to describe a two-flavor nonlocal PNJL model at finite temperature and nonvanishing isospin chemical potential. In Sec. III we quote and discuss our numerical results, including the comparison with the outcomes from alternative effective approaches and LQCD simulations. Finally, in Sec. IV we summarize our results and present our main conclusions.

\section{THEORETICAL FORMALISM}

We start by considering the Euclidean action of a twoflavor quark model that includes nonlocal scalar and pseudoscalar quark-antiquark currents. One has

$S_{E}=\int d^{4} x\left[\bar{\psi}(x)(-i \not \partial+\hat{m}) \psi(x)-\frac{G}{2} j_{a}(x) j_{a}(x)\right]$,

where $\psi=\left(\psi_{u} \psi_{d}\right)^{T}$ stands for the $u, d$ quark-field doublet, and $\hat{m}=\operatorname{diag}\left(m_{u}, m_{d}\right)$ is the current quark-mass matrix. In what follows we assume that the current masses of $u$ and $d$ quarks are equal, denoting $m_{c} \equiv m_{u}=m_{d}$. The nonlocal currents $j_{a}(x)$ in Eq. (1) are given by

$$
j_{a}(x)=\int d^{4} z \mathcal{G}(z) \bar{\psi}\left(x+\frac{z}{2}\right) \Gamma_{a} \psi\left(x-\frac{z}{2}\right),
$$

where we have defined $\Gamma_{a}=\left(\mathbb{1}, i \gamma_{5} \vec{\tau}\right)$ with $\tau_{i}$ being Pauli matrices that act on flavor space. The function $\mathcal{G}(z)$ is a form factor responsible for the nonlocal character of the four-point interactions.

To study strong-interaction matter at finite temperature and/or chemical potential we introduce the partition function of the system, given by $\mathcal{Z}=\int \mathcal{D} \bar{\psi} \mathcal{D} \psi \exp \left[-S_{E}\right]$. As stated, we are interested in dealing with isospin asymmetric matter. This is effectively implemented by introducing quark chemical potentials $\mu_{u}$ and $\mu_{d}$, which in principle 
can be different from each other. Thus, in the effective action we perform the replacement

$$
\left(\begin{array}{cc}
\partial_{4} & 0 \\
0 & \partial_{4}
\end{array}\right) \rightarrow\left(\begin{array}{cc}
\partial_{4}-\mu_{u} & 0 \\
0 & \partial_{4}-\mu_{d}
\end{array}\right) .
$$

The quark chemical potentials can be written in terms of average and isospin chemical potentials $\mu$ and $\mu_{I}$ as

$$
\mu_{u}=\mu+\frac{\mu_{I}}{2}, \quad \mu_{d}=\mu-\frac{\mu_{I}}{2},
$$

where $\mu=\mu_{B} / 3$, with $\mu_{B}$ being the baryon chemical potential. For the nonlocal model under consideration, to obtain the appropriate conserved currents the replacement in Eq. (3) has to be complemented with a modification of the nonlocal currents appearing in Eq. (2), namely [53,54]

$$
\begin{aligned}
\psi(x-z / 2) & \rightarrow \mathcal{W}(x, x-z / 2) \psi(x-z / 2), \\
\bar{\psi}(x+z / 2) & \rightarrow \bar{\psi}(x+z / 2) \gamma_{0} \mathcal{W}(x+z / 2, x) \gamma_{0} .
\end{aligned}
$$

In the present case the transport functions $\mathcal{W}$ are simply given by

$$
\mathcal{W}(x, x-z / 2)=\mathcal{W}(x+z / 2, x)=\exp \left(\frac{z_{4}}{2} \hat{\mu}\right),
$$

where $\hat{\mu}=\operatorname{diag}\left(\mu_{u}, \mu_{d}\right)$.

It is convenient to perform a standard bosonization of the fermionic action [55], introducing auxiliary mesonic fields $\sigma$ and $\pi_{i}, i=1,2,3$, and integrating out the fermion fields. We consider here the mean field approximation (MFA), in which the bosonic fields are replaced by their vacuum expectation values (VEVs), $\bar{\sigma}$ and $\bar{\pi}_{i}$. Though this is a widely used approach, it is important to keep in mind that quantum fluctuations may be significant for a precise determination of the behavior of thermodynamic quantities and the features of the phase diagram. Estimations of these effects have been studied taking into account the functional renormalization group and the presence of mesonic fluctuations, in the framework of the NJL and the quark-meson models (see e.g., Refs. [34,56-59]). It can be seen that fluctuations lead to a softening of the chiral restoration transition and tend to shift to lower temperatures the critical end point in the $T-\mu_{B}$ phase diagram.

Let us recall that, for $\mu_{I}=0$, in the chiral limit $\left(m_{c}=0\right)$ the action in Eq. (1) is invariant under global $\mathrm{U}(1)_{B} \otimes$ $\mathrm{SU}(2)_{I} \otimes \mathrm{SU}(2)_{I A}$ transformations. The group $\mathrm{U}(1)_{B}$ is associated to baryon number conservation, while the chiral group $\mathrm{SU}(2)_{I} \otimes \mathrm{SU}(2)_{I A}$ corresponds to the symmetries under isospin and axial-isospin transformations. At zero temperature the $\mathrm{SU}(2)_{I A}$ symmetry is expected to be spontaneously broken by a large value of $\bar{\sigma}$ (which leads to large constituent quark masses), while at high temperatures one expects to have $\bar{\sigma}=0$, which implies a restoration of the chiral symmetry. In the presence of finite quark masses one has an explicit breakdown of $\mathrm{SU}(2)_{I A}$ (and also of SU(2) $)_{I}$, if current $u$ and $d$ quark masses are different to each other), hence the chiral symmetry is expected to be only partially restored at high $T$. Now, in the presence of a nonzero isospin chemical potential the full chiral symmetry group is explicitly broken down to the $\mathrm{U}(1)_{I_{3}} \otimes \mathrm{U}(1)_{I_{3} A}$ subgroup. At $T=0$ it might happen that, similarly to the $\mu_{I}=0$ case, $\mathrm{U}(1)_{I_{3} A}$ is spontaneously broken by a large value of $\bar{\sigma}$. Moreover, while for finite current quark masses one has $\bar{\pi}_{3}=0$ [60], it can happen that nonvanishing VEVs for $\pi_{1}$ and $\pi_{2}$ be developed, leading to a spontaneous breakdown of the remaining $\mathrm{U}(1)_{I_{3}}$ symmetry. Since the action is still invariant under $\mathrm{U}(1)_{I_{3}}$ transformations, without loss of generality one can choose $\bar{\pi}_{i}=\delta_{i 1} \bar{\Delta}$.

We consider the above described general situation in which both $\bar{\sigma}$ and $\bar{\Delta}$ can be nonvanishing. At zero temperature, the mean field thermodynamic potential is found to be given by

$$
\begin{aligned}
\Omega^{\mathrm{MFA}}(T=0)= & \frac{\bar{\sigma}^{2}+\bar{\Delta}^{2}}{2 G} \\
& -\operatorname{Tr} \ln \left(\begin{array}{cc}
\not \not_{u}+M\left(p_{u}\right) & i \gamma_{5} \rho(\bar{p}) \\
i \gamma_{5} \rho(\bar{p}) & \not p_{d}+M\left(p_{d}\right)
\end{array}\right),
\end{aligned}
$$

where

$$
M(p)=m_{c}+g(p) \bar{\sigma}, \quad \rho(p)=g(p) \bar{\Delta} .
$$

Here we have defined $p_{f}^{\nu} \equiv\left(\vec{p}, p_{4}+i \mu_{f}\right)$, with $f=u, d$, and $\bar{p}=\left(p_{u}+p_{d}\right) / 2$. The function $g(p)$ is the Fourier transform of the form factor $\mathcal{G}(z)$ in Eq. (2).

Let us consider the extension of the model to the case of finite temperature, which can be addressed by using the standard Matsubara formalism. In order to account for confinement effects, we include the coupling of fermions to the Polyakov loop (PL). This is done by replacing the derivative in the kinetic term in Eq. (1) by the covariant derivative $\partial_{\mu}-i g \lambda_{a} G_{\mu a} / 2$, where $G_{\mu a}$ are $\mathrm{SU}(3)$ color gauge fields, and assuming that quarks move on a uniform color background field $\phi=g G_{4 a} \lambda_{a} / 2$. Neglecting spacial components of gluon fields, we consider the so-called Polyakov gauge, in which $G_{4 a}$ is static $\left(\partial G_{4 a} / \partial x_{4}=0\right)$ and $\phi$ can be rotated to a diagonal representation $\phi=\operatorname{diag}\left(\phi_{r}, \phi_{g}, \phi_{b}\right)=\phi_{3} \lambda_{3}+\phi_{8} \lambda_{8}$. In this framework, we take the traced Polyakov loop $\Phi=\frac{1}{3} \operatorname{Tr} \exp (i \phi / T)$ as an order parameter of the confinement/deconfinement transition. In addition, to account for effective gauge field selfinteractions we introduce a mean field Polyakov-loop potential $\mathcal{U}$ that depends on the traced PL, its conjugate $\bar{\Phi}$ and the temperature. The resulting scheme is referred to as a nonlocal Polyakov-loop extended Nambu-Jona-Lasinio model [29-34].

Concerning the PL potential, its functional form can be proposed on the basis of pure gauge QCD properties. Let us 
consider a potential given by a polynomial function based on a Ginzburg-Landau ansatz $[39,61]$, namely

$\frac{\mathcal{U}_{\mathrm{poly}}(\Phi, \bar{\Phi}, T)}{T^{4}}=-\frac{b_{2}(T)}{2} \bar{\Phi} \Phi-\frac{b_{3}}{6}\left(\Phi^{3}+\bar{\Phi}^{3}\right)+\frac{b_{4}}{4}(\bar{\Phi} \Phi)^{2}$,

where

$b_{2}(T)=a_{0}+a_{1}\left(\frac{T_{0}}{T}\right)+a_{2}\left(\frac{T_{0}}{T}\right)^{2}+a_{3}\left(\frac{T_{0}}{T}\right)^{3}$
The parameters $a_{i}$ and $b_{i}$ can be fitted to pure gauge lattice QCD results imposing the presence of a first-order phase transition at the reference temperature $T_{0}$, which is a further parameter of the model. In the absence of dynamical quarks, $T_{0}$ is the critical temperature for deconfinement, and from lattice QCD calculations one expects it to be approximately equal to $270 \mathrm{MeV}$. However, it has been argued that in the presence of light dynamical quarks $T_{0}$ should be rescaled to about $210 \mathrm{MeV}$ and $190 \mathrm{MeV}$ for the case of two and three flavors, respectively, with an uncertainty of about $30 \mathrm{MeV}[62,63]$. The numerical values for the PL potential parameters are [39]

$$
a_{0}=6.75, \quad a_{1}=-1.95, \quad a_{2}=2.625, \quad a_{3}=-7.44, \quad b_{3}=0.75, \quad b_{4}=7.5 .
$$

As an alternative form for the PL potential, often used in the literature, one can take a logarithmic expression based on the Jacobi determinant that arises from the integration of the nondiagonal piece of the SU(3) color group [40]. The effect of choosing this alternative form is discussed in Sec. III B.

After the inclusion of PL interactions, the grand canonical thermodynamic potential of the system is given by

$$
\begin{aligned}
\Omega^{\mathrm{MFA}}= & \frac{\bar{\sigma}^{2}+\bar{\Delta}^{2}}{2 G}-2 T \sum_{n=-\infty}^{\infty} \sum_{c=r, g, b} \int \frac{d^{3} p}{(2 \pi)^{3}} \ln \left\{E_{n u c}^{2} E_{n d c}^{2}\right. \\
& \left.-\rho\left(\bar{p}_{n c}\right)^{2}\left[\left(M\left(p_{n u c}\right)-M\left(p_{n d c}\right)\right)^{2}-\left(\mu_{u}-\mu_{d}\right)^{2}\right]\right\}+\mathcal{U}_{\text {poly }}(\Phi, \bar{\Phi}, T),
\end{aligned}
$$

where we have introduced the definitions $\bar{p}_{n c}=\left(p_{n u c}+p_{n d c}\right) / 2$ and $E_{n f c}^{2}=M\left(p_{n f c}\right)^{2}+p_{n f c}^{2}+\rho\left(\bar{p}_{n c}\right)^{2}$, with $p_{n f c} \equiv\left(\vec{p},(2 n+1) \pi T+i \mu_{f}-\phi_{c}\right)$. As usual in this type of model, it is seen that $\Omega^{\mathrm{MFA}}$ turns out to be divergent, thus it has to be regularized. We adopt here a prescription similar as the one considered e.g., in Ref. [64], viz.

$$
\Omega^{\mathrm{MFA}, \text { reg }}=\Omega^{\mathrm{MFA}}-\Omega_{\mathrm{q}}^{\text {free }}+\Omega_{\mathrm{q}}^{\text {free,reg }}+\Omega_{0} .
$$

Here the "free" potential keeps the interaction with the PL, while $\bar{\sigma}$ and $\bar{\Delta}$ are set to zero. A constant term $\Omega_{0}$ is also added so as to fix $\Omega^{\mathrm{MFA} \text {,reg }}=0$ at $\mu_{B}=\mu_{I}=T=0$. For the regularized form of the free piece, the Matsubara sum can be performed analytically. One has

$$
\Omega_{\mathrm{q}}^{\text {free,reg }}=-2 T \sum_{f=u, d} \sum_{c=r, g, b} \sum_{s= \pm 1} \int \frac{d^{3} \vec{p}}{(2 \pi)^{3}} \operatorname{Re} \ln \left[1+\exp \left(-\frac{\epsilon_{f}+s\left(\mu_{f}+i \phi_{c}\right)}{T}\right)\right],
$$

where $\epsilon_{f}=\sqrt{\vec{p}^{2}+m_{f}^{2}}$.

The mean field values $\bar{\sigma}$ and $\bar{\Delta}$, as well as the values of $\phi_{3}$ and $\phi_{8}$, can now be obtained from a set of four coupled "gap equations" that follow from the minimization of the regularized thermodynamic potential, namely

$$
\frac{\partial \Omega^{\mathrm{MFA}, \mathrm{reg}}}{\partial \bar{\sigma}}=0, \quad \frac{\partial \Omega^{\mathrm{MFA}, \mathrm{reg}}}{\partial \bar{\Delta}}=0, \quad \frac{\partial \Omega^{\mathrm{MFA}, \mathrm{reg}}}{\partial \phi_{3}}=0, \quad \frac{\partial \Omega^{\mathrm{MFA}, \mathrm{reg}}}{\partial \phi_{8}}=0 .
$$

In addition, it is interesting to study the behavior of quark condensates. As usual, we consider the scalar condensate $\Sigma=\Sigma_{u}+\Sigma_{d}$, where $\Sigma_{f}=\left\langle\bar{\psi}_{f} \psi_{f}\right\rangle$. The corresponding expressions can be obtained by differentiating $\Omega^{\mathrm{MFA}, \text { reg }}$ with respect to the current up and down current quark masses, i.e.,

$$
\Sigma_{f}=\frac{\partial \Omega^{\mathrm{MFA}, \mathrm{reg}}}{\partial m_{f}}
$$


Another relevant quantity is the charged pion condensate $\Pi$, which is expected to be nonvanishing for $\mu_{I} \neq 0$. According to our choice $\bar{\pi}_{i}=\delta_{i 1} \bar{\Delta}$, we get

$$
\Pi=\left\langle\bar{\psi} i \gamma_{5} \tau_{1} \psi\right\rangle .
$$

The analytical expression for this condensate can be obtained by taking the derivative of the regularized thermodynamic potential with respect to an auxiliary parameter added to $\rho(\bar{p})$ in Eq. (7), and then set to zero after the calculation.

To study the phase transitions, we also introduce the susceptibilities associated to the $\Sigma$ and $\Pi$ condensates [65] and the Polyakov loop. These are given by

$$
\chi_{\mathrm{ch}}=-\frac{\partial \Sigma}{\partial m_{c}}, \quad \chi_{\Pi}=\frac{\partial \Pi}{\partial m_{c}}, \quad \chi_{\Phi}=\frac{d \Phi}{d T} .
$$

Finally, from the regularized potential one can calculate various thermodynamic quantities, such as the pressure $p$, the energy and entropy densities $\varepsilon$ and $s$, and the particle number densities $n_{I}$ and $n_{B}$. The corresponding expressions are

$$
\begin{aligned}
p & =-\Omega^{\mathrm{MFA}, \mathrm{reg}}, \\
\varepsilon & =\Omega^{\mathrm{MFA}, \mathrm{reg}}+T s+n_{I} \mu_{I}+n_{B} \mu_{B}, \\
s & =-\frac{\partial \Omega^{\mathrm{MFA}, \mathrm{reg}}}{\partial T}, \\
n_{I} & =-\frac{\partial \Omega^{\mathrm{MFA}, \mathrm{reg}}}{\partial \mu_{I}}, \\
n_{B} & =-\frac{\partial \Omega^{\mathrm{MFA}, \mathrm{reg}}}{\partial \mu_{B}} .
\end{aligned}
$$

In this work we restrict to the case of $\mu_{B}=0$, focusing on the effect of finite isospin chemical potential $\mu_{I}$. As stated in the Introduction, in this situation the results from effective models can be compared with existing lattice QCD calculations [25,26,66-68], which do not suffer from the sign problem. Since the thermodynamic potential turns out to be real, one gets $\Phi=\bar{\Phi}, \phi_{8}=0$, and the last of Eqs. (15) is trivially satisfied.

\section{NUMERICAL RESULTS}

To fully define the model it is necessary to specify the form factor entering the nonlocal fermion current in Eq. (2). In this work we consider an exponential momentum dependence for the form factor in momentum space,

$$
g(p)=\exp \left(-p^{2} / \Lambda^{2}\right)
$$

This form, which is widely used, guarantees a fast ultraviolet convergence of quark loop integrals. Notice that the energy scale $\Lambda$, which acts as an effective momentum cutoff, has to be taken as an additional parameter of the model. Other functional forms, e.g., Lorentzian form factors with integer [54] or fractional [69] momentum dependences, have also been considered in the literature. In any case, it is seen that the form factor choice does not have in general major impact in the qualitative predictions for the relevant thermodynamic quantities [70].

Given the form factor shape, the model parameters $m_{c}, G$, and $\Lambda$ can be fixed by requiring that the model reproduce the phenomenological values of some selected physical quantities. If we take as inputs the pion mass $m_{\pi}=138 \mathrm{MeV}$, the pion weak decay constant $f_{\pi}=92.4 \mathrm{MeV}$ and the quark condensates $\Sigma_{u}=\Sigma_{d}=-(240 \mathrm{MeV})^{3}$, one has $m_{c}=$ 5.67 MeV, $\Lambda=752 \mathrm{MeV}$, and $g=G \Lambda^{2}=20.67$ [53].

\section{A. Zero temperature}

At zero temperature the Polyakov loop decouples from the fermions, and the thermodynamic potential within the nonlocal NJL (nlNJL) model is given by the expression in Eq. (7), properly regularized. In Fig. 1 we show our numerical results for the normalized mean field condensates $\Sigma / \Sigma_{0}$ and $\Pi / \Sigma_{0}$, where $\Sigma_{0} \equiv \Sigma\left(\mu_{I}=0\right)$, as functions of the isospin chemical potential. The solid red lines correspond to the parametrization described above, which leads to $\Sigma_{0}=-(240 \mathrm{MeV})^{3}$. To provide an estimation of the parametrization dependence, we show with a redshaded band the results covered by a parameter range such that $\Sigma_{0}$ lies between $-(230 \mathrm{MeV})^{3}$ and $-(250 \mathrm{MeV})^{3}$. The right panel of Fig. 1 just extends the results given in the left panel, covering a broader range of values of the scaled isospin chemical potential $\mu_{I} / m_{\pi}$. For comparison, in both panels we include the results obtained from several alternative approaches. The green band (partially hidden by the red one) corresponds to the results from the local NJL, for parametrizations leading to a quark condensate in the range between $-(240 \mathrm{MeV})^{3}$ and $-(250 \mathrm{MeV})^{3}$. The dashed (green) lines, the dotted (brown) lines and the dashed-dotted (blue) lines correspond to the results obtained within the linear sigma model (LSM) in Ref. [46], the NJL model in Ref. [47] (where a medium separation regularization scheme is used) and the chiral perturbation theory (ChPT) approach in Ref. [51], respectively. In addition, the fat dots denote the results from lattice QCD obtained in Ref. [26].

As expected, for $\mu_{I}<m_{\pi}$ one has $\Sigma=\Sigma_{0}$ and $\Pi=0$. Indeed, for both local and nonlocal NJL models it can be analytically shown that the onset of the pion condensation at $T=0$ occurs at $\mu_{I}=m_{\pi}$. For larger isospin chemical potentials, as shown in Fig. 1, the chiral condensate decreases monotonically and the charged pion condensate gets strongly increased. In this way, for $\mu_{I} \geq m_{\pi}$ the isospin symmetry $\mathrm{U}(1)_{I_{3}}$ gets spontaneously broken, while one finds a partial restoration of the $\mathrm{U}(1)_{I_{3} A}$ symmetry for large values of $\mu_{I}$. From the left panel of Fig. 1 it is also seen that there is an overall agreement between most theoretical 

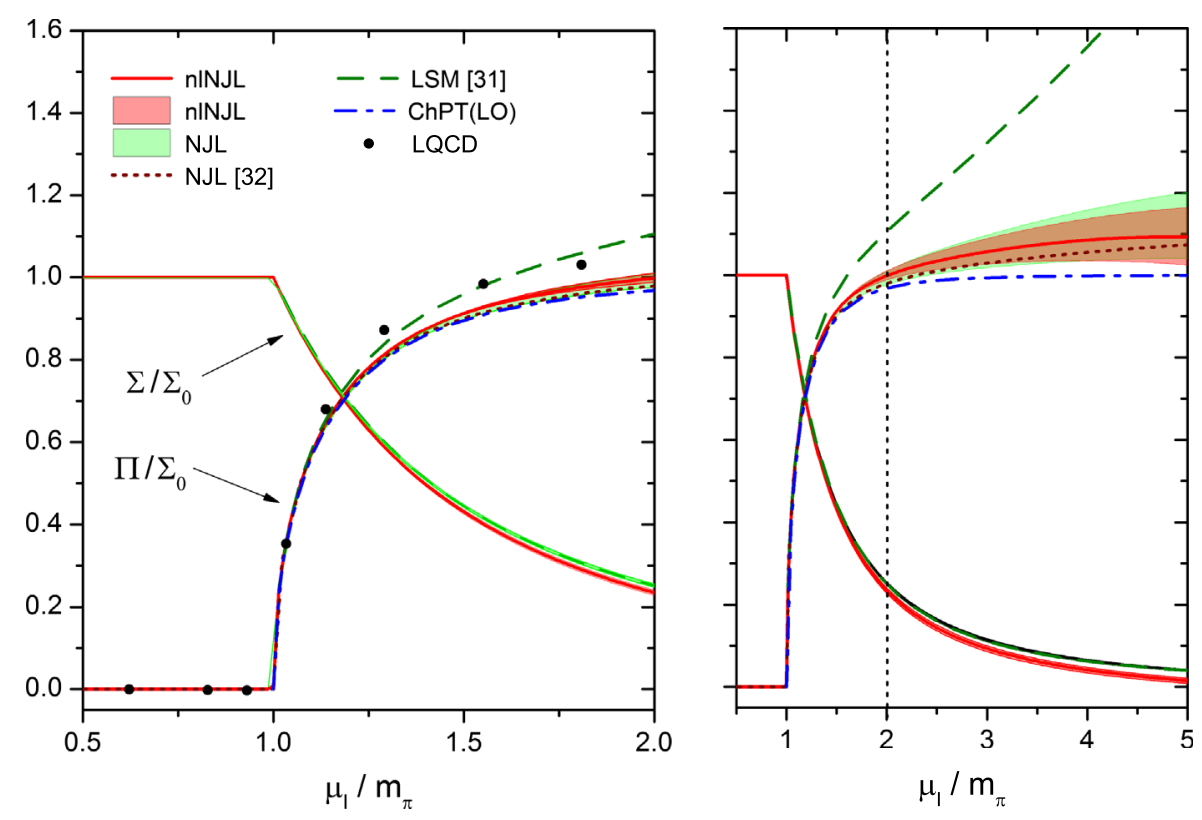

FIG. 1. (Color online) Normalized $\Sigma$ and $\Pi$ condensates as functions of the isospin chemical potential. The solid red line and the red band correspond to the numerical results obtained within the nINJL model. Results from other theoretical approaches (see text) are included for comparison.

approaches up to $\mu_{I} \simeq 2 m_{\pi}$. On the other hand, as shown in the right panel of the figure, for larger values of $\mu_{I}$ there is some splitting between the predictions from different models.

The results for the chiral and pion condensate susceptibilities as functions of $\mu_{I}$ are displayed in Fig. 2. It can be seen that the chiral susceptibility $\chi_{\mathrm{ch}}$ (solid line, left panel) is approximately zero for low values of $\mu_{I}$, showing a jump to a high value at $\mu_{I}=m_{\pi}$ and remaining relatively large for $\mu_{I}>m_{\pi}$. This signals that at $\mu_{I}=m_{\pi}$ one has the onset of a smooth transition from a phase in which the $\mathrm{U}(1)_{I_{3} A}$ symmetry is spontaneously broken to a region in which it becomes (partially) restored. It is found that the height of

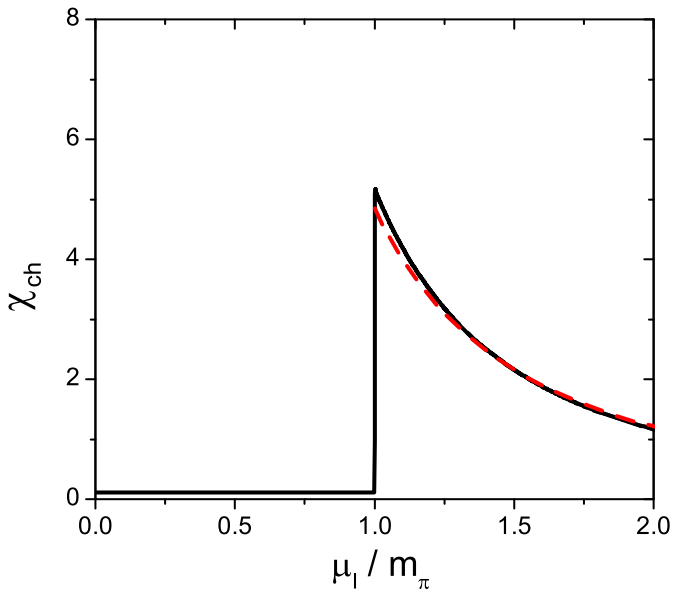

the jump at $\mu_{I}=m_{\pi}$ gets increased if the current quark mass $m_{c}$ is reduced. The pion condensate susceptibility is given by the solid line in the right panel of Fig. 2. It is seen that $\chi_{\Pi}$ is zero for low values of $\mu_{I}$, and has a divergence at $\mu_{I}=m_{\pi}$. This is the signature of a second-order phase transition leading to the appearance of the pion condensate, as shown in Fig. 1. The behavior of the susceptibilities is similar to the one found in the local NJL model, see Ref. [65].

It is interesting to compare the above results with those arising from chiral perturbation theory. At the lowest order in the chiral expansion, it is found that for $\mu_{I} \geq m_{\pi}$ the condensates satisfy the relations [71]

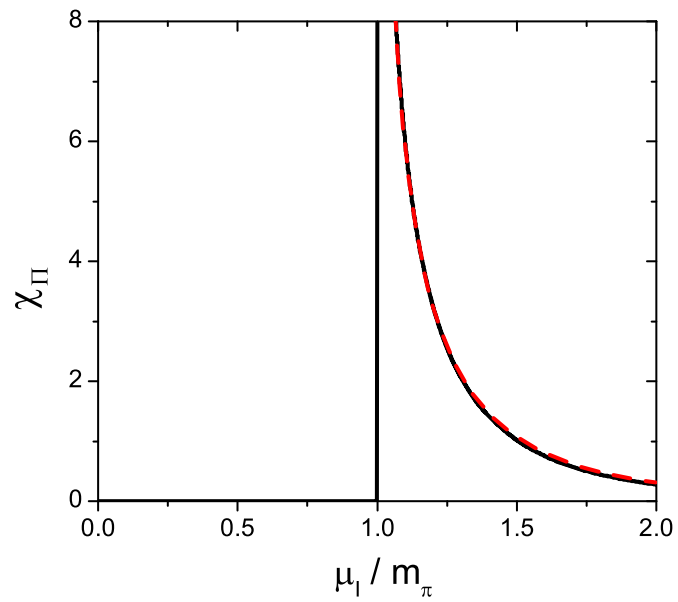

FIG. 2. (Color online) Chiral and pion susceptibilities as functions of the isospin chemical potential. Solid and dashed lines correspond to the results from nINJL model calculations and lowest-order ChPT expressions, respectively. 

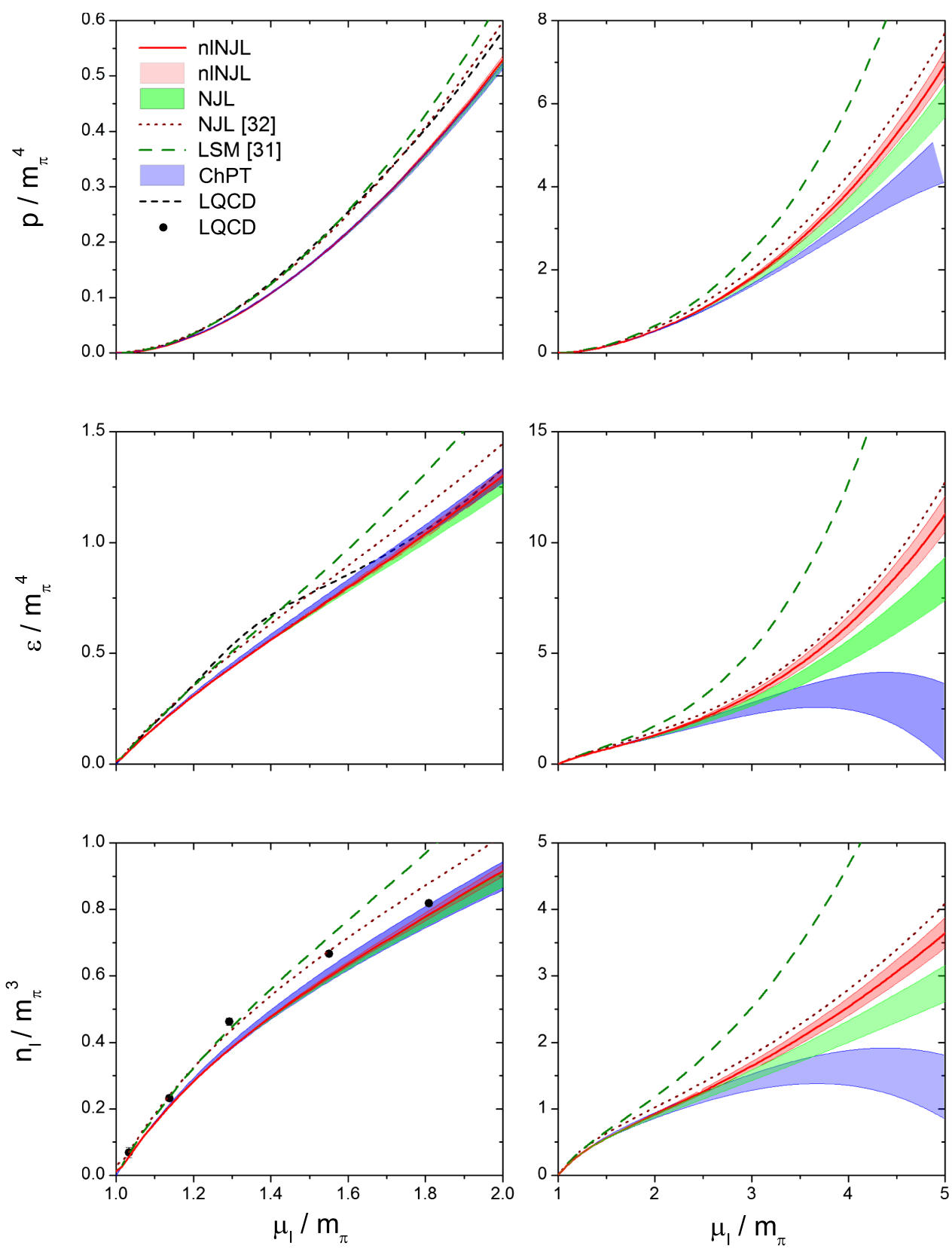

FIG. 3. (Color online) Numerical results for the normalized pressure, energy density and isospin particle density as functions of the isospin chemical potential. Besides the local and nonlocal NJL models, the graphs include the results obtained from the ChPT approach in Ref. [51], the linear sigma model in Ref. [46], and LQCD calculations in Refs. [26,47].

$$
\frac{\Sigma}{\Sigma_{0}}=\frac{m_{\pi}^{2}}{\mu_{I}^{2}}, \quad \frac{\Pi}{\Sigma_{0}}=\sqrt{1-\frac{m_{\pi}^{2}}{\mu_{I}^{2}}} .
$$

In this way one has

$$
\left(\frac{\Sigma}{\Sigma_{0}}\right)^{2}+\left(\frac{\Pi}{\Sigma_{0}}\right)^{2}=1
$$

which defines the so-called "chiral circle". The relation in Eq. (22) is approximately satisfied in local and nonlocal
NJL models, as can be seen from Fig. 1. In fact, the agreement is very good up to $\mu_{I} \simeq 2 m_{\pi}$, where the prediction from ChPT is trustable. Moreover, with the aid of the Gell-Mann-Oakes-Renner relation one can find simple analytical expressions for the susceptibilities, namely

$$
\chi_{\mathrm{ch}}=-\frac{\Sigma_{0}}{m_{c}} \frac{m_{\pi}^{2}}{\mu_{I}^{2}}, \quad \chi_{\Pi}=-\frac{\Sigma_{0}}{m_{c}} \frac{m_{\pi}^{4}}{\mu_{I}^{4}} \frac{1}{\sqrt{1-\frac{m_{\pi}^{4}}{\mu_{I}^{4}}}},
$$



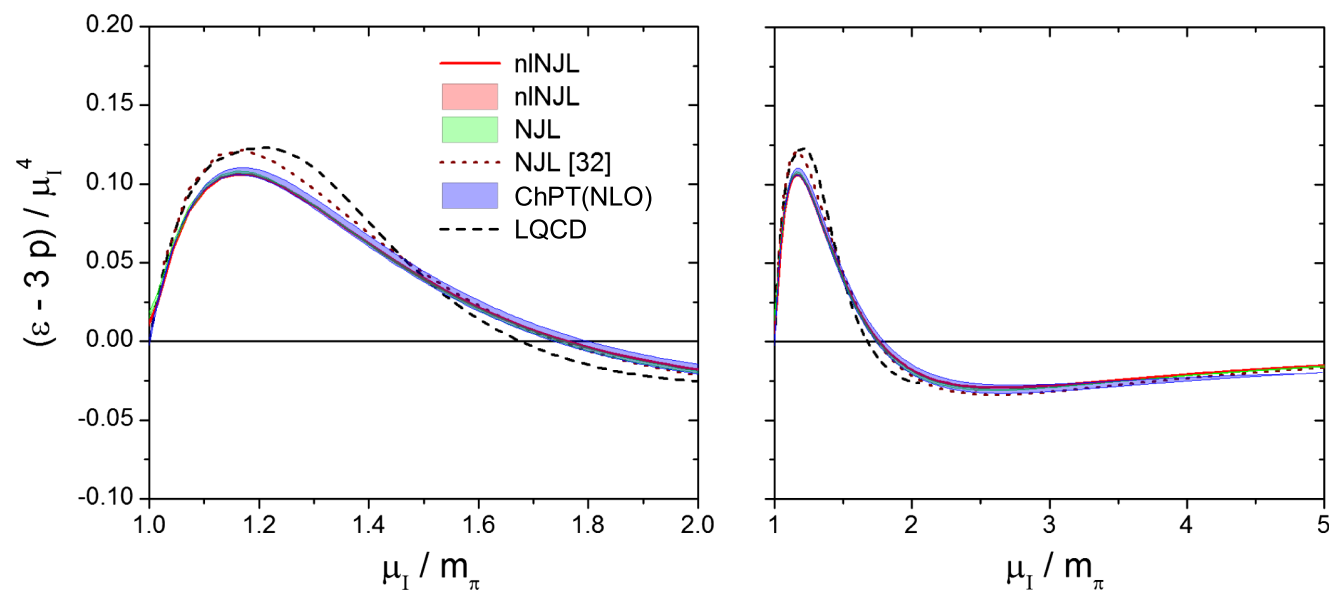

FIG. 4. (Color online) Numerical results for the interaction energy as function of the isospin chemical potential. The graphs include the values obtained from local and nonlocal NJL models, ChPT [51] and LQCD calculations [26,47].

where it has been assumed that the ratio $\Sigma_{0} / f_{\pi}^{2}$ is approximately independent of $m_{c}$. In Eqs. (23), it can be seen that $\chi_{\Pi}$ diverges at $\mu_{I}=m_{\pi}$, while $\chi_{\mathrm{ch}}$ is finite and only becomes divergent in the chiral limit. The behavior of the susceptibilities as functions of $\mu_{I}$ obtained from these equations are shown by the dashed lines in Fig. 2. It can be seen that they match nicely the results arising from the nlNJL model.

Next, in Fig. 3 we show the results obtained within the nlNJL model for the normalized pressure, energy density, and isospin particle density as functions of $\mu_{I} / m_{\pi}$. Results from other theoretical approaches are also included for comparison (lines and bands for NJL and nINJL models are defined in the same way as in Fig. 1). In the left panels we consider a range of $\mu_{I}$ from $m_{\pi}$ to $2 m_{\pi}$, for which LQCD estimations have been obtained in Refs. [26,47] (shortdashed black lines and fat dots in the figure). In the right panels we include the results for the same quantities using a different scale that covers values of the isospin chemical potential up to $5 m_{\pi}$. Notice that all three quantities are zero for $0 \leq \mu_{I} \leq m_{\pi}$. From the left panels it can be seen that in general there is a good agreement between the predictions of effective models-which do not differ significantly from each other-and LQCD results. On the other hand, for larger values of $\mu_{I}$ the splitting between the results from various theoretical approaches becomes appreciably large. Unfortunately, no LQCD results are currently available within this enlarged range. The behavior of the studied quantities for the nonlocal approach (solid red lines, red bands) is found to be qualitatively similar to the one obtained within the local NJL model (green bands), showing a monotonic growth when $\mu_{I}$ gets increased. Notice that the dependence on the parametrization turns out to be relatively low.

Another interesting magnitude to be analyzed is the interaction energy, or trace anomaly, $\epsilon-3 p$. The behavior of this quantity (normalized by $\mu_{I}^{4}$ ) as a function of $\mu_{I} / m_{\pi}$, is shown in Fig. 4. It is seen that the results obtained within
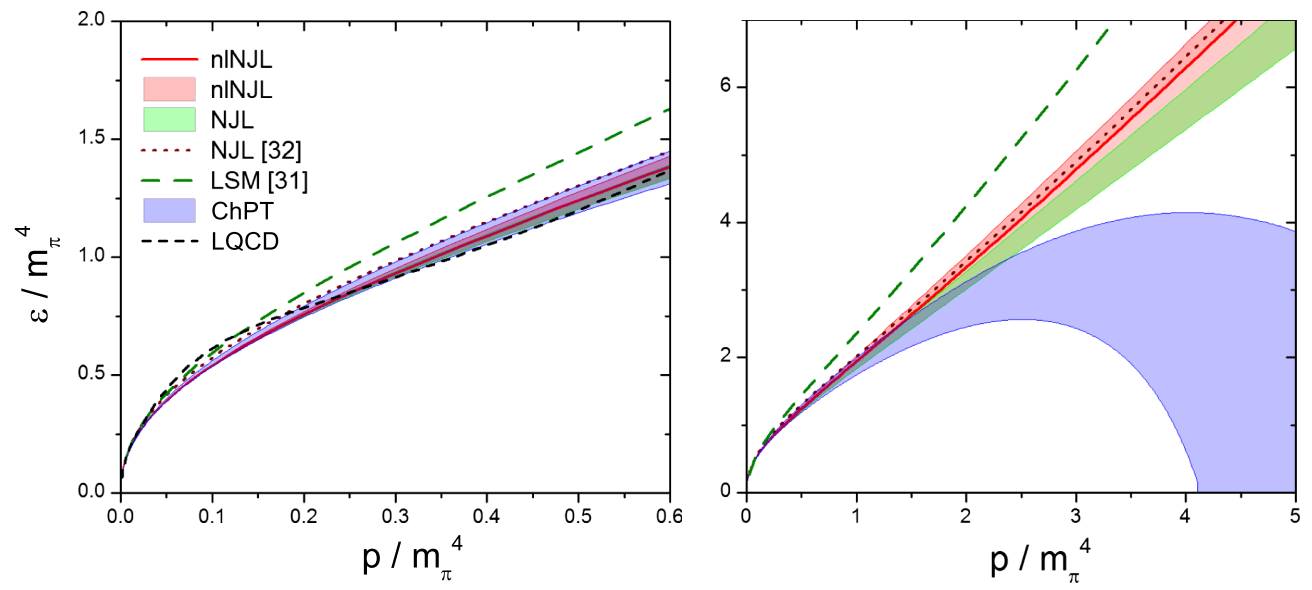

FIG. 5. (Color online) Numerical results for the equation of state. The graphs include the results obtained from local and nonlocal NJL models, the linear sigma model [46], ChPT [51] and LQCD calculations [26,47]. 
the nlNJL model are similar to those found in other theoretical approaches. In particular, the so-called "conformal point", for which $\epsilon=3 p$, is reached at a value of $\mu_{I} / m_{\pi}$ in the range between 1.75 and 1.77 (depending on the parametrization), in good agreement with the analytical result $\mu_{I} / m_{\pi}=\sqrt{3}$ arising from leading-order ChPT [72].

To conclude this subsection, in Fig. 5 we plot the numerical results obtained for the equation of state, i.e., the behavior of the energy density as a function of the pressure (here the isospin chemical potential $\mu_{I}$ is an underlying parameter). The notation for the curves obtained within the nonlocal NJL approach and other models, as well as those arising from lattice QCD calculations, are the same as in Figs. 3 and 4. Once again, the results from the nonlocal approach are qualitatively similar to those obtained in the local NJL model, and are consistent with LQCD results in the low-energy region (where LQCD data are available).

\section{B. Finite temperature}

We present here our numerical results at finite temperature for the quantities defined in Sec. II. As discussed above, we include the interaction between the fermions and a background color field, considering the Polyakov loop potential in Eq. (9). The parameter $T_{0}$ entering this potential is taken to be $200 \mathrm{MeV}$, following the estimations carried out for the case of two dynamical quarks $[62,63]$.

Let us start by studying the thermal behavior of the normalized mean field condensates and the traced PL for some representative values of $\mu_{I}$ within the range $0 \leq \mu_{I} \leq 2 m_{\pi}$. Our results are shown in Fig. 6. On the left panels we plot the condensates $\Sigma$ and $\Pi$, normalized by $\Sigma_{0}$ (solid and dashed lines, respectively), together with the traced PL $\Phi$ (dashed-dotted lines). The results are given as functions of the temperature, normalized to the critical temperature for $\mu_{I}=0$, viz. $T_{c}^{0}=174 \mathrm{MeV}$. We also include the curves for the normalized combined quantity $R$, defined by

$$
R=\frac{\sqrt{\Sigma^{2}+\Pi^{2}}}{\Sigma_{0}} .
$$

In the right panels of Fig. 6 we plot the susceptibilities associated to the chiral and pion condensates and the traced Polyakov loop (solid, dashed, and dashed-dotted lines, respectively), defined in Eq. (18). As usual, the peaks of the curves for $\chi_{\mathrm{ch}}$ and $\chi_{\Phi}$ are used in order to define the chiral restoration and deconfinement transition critical temperatures.

From the left panels of Fig. 6 it is seen that for $\mu_{I}=0$ the chiral restoration and deconfinement transitions proceed as a smooth crossovers at temperatures $T \simeq T_{c}^{0}$, while the pion condensate vanishes for all $T$. The situation remains basically the same up to values of $\mu_{I}$ approaching $m_{\pi}$. Then, for a small region of values of $\mu_{I}$ just below $m_{\pi}$ (as shown explicitly for the case of $\left.\mu_{I} / m_{\pi}=0.99\right)$ the pion condensate vanishes for all $T$ except for a short range of temperatures slightly below the critical value $T_{c}$ that characterizes the (almost simultaneous) chiral restoration and deconfinement crossover transitions. On the other hand, for $\mu_{I}>m_{\pi}$, at low temperatures the pion condensate gets nonzero values, showing the spontaneous breakdown of isospin symmetry. These values of $\Pi$ are approximately independent of the temperature up to $T \simeq T_{c}^{0}$, where one finds a second-order transition to a $\mathrm{U}(1)_{I_{3}}$ symmetry restored phase. In addition, it can be seen that these values of $\Pi$ get increased with $\mu_{I}$, while the values of the chiral condensate $\Sigma$ decrease, in such a way that $R$ is approximately constant. We recall that, as discussed in the previous subsection, from lowest-order ChPT one gets at $T=0$ a constant value $R=1$ for all values of $\mu_{I}$. Moreover, as noted in Ref. [46], the behavior of $R$ as a function of $T$ is very similar to that found for $\Sigma / \Sigma_{0}$ when pion condensation is not considered. Concerning the deconfinement transition, the graphs on the left panel of Fig. 6 show that it proceeds as a smooth crossover at an approximately constant temperature $T \lesssim T_{c}^{0}$ for the considered range of values of the isospin chemical potential.

Taking now into account the plots in the right panels of Fig. 6, it can be seen that the PL susceptibility (green dashed-dotted lines) shows clear peaks that indicate a crossover-like deconfinement transition at a temperature slightly lower than $T_{c}^{0}$ and approximately independent of $\mu_{I}$. In the case of the chiral susceptibility (red solid lines in the right panels of Fig. 6), for $\mu_{I}=0$ one finds a peak that defines the critical temperature, $T_{c}^{0}=174 \mathrm{MeV}$. Notice that for $\mu_{I}$ larger than $m_{\pi}$ the susceptibility $\chi_{\mathrm{ch}}$ is relatively large at low temperatures. This is in agreement with the behavior shown in Fig. 2, and it can be attributed to the presence of a nonzero pion condensate. The same effect occurs for values of $\mu_{I}$ slightly below $m_{\pi}$ and temperatures $T \lesssim T_{c}^{0}$, owing to the existence of the already mentioned nonzero value of $\Pi$ in this region (see the panels of the second row in Fig. 6). Finally, the pion condensate susceptibility (dashed lines in the right panels of Fig. 6) is also found to be nonzero in the presence of the pion condensate. Moreover, as expected, it becomes divergent at the temperatures in which one finds the second-order phase transition into the isospin symmetry restored phase. These temperatures are slightly lower than $T_{c}^{0}$ and basically coincide with the ones corresponding to the deconfinement transition. For completeness, we show in Fig. 7 the behavior of the $\Sigma$ and $\Pi$ susceptibilities as functions of the isospin chemical potential, for $T=0$ and temperatures slightly below and above $T_{c}^{0}$. In fact, it is seen that the behavior of $\chi_{\mathrm{ch}}$ and $\chi_{\Pi}$ found for $T=0$ (see Fig. 2) does not change qualitatively up to the critical isospin symmetry restoration temperature. Notice that for temperatures just below $T_{c}^{0}$ the position of the discontinuity is shifted to $\mu_{I} / m_{\pi}$ slightly smaller than one. It is also worth noticing 

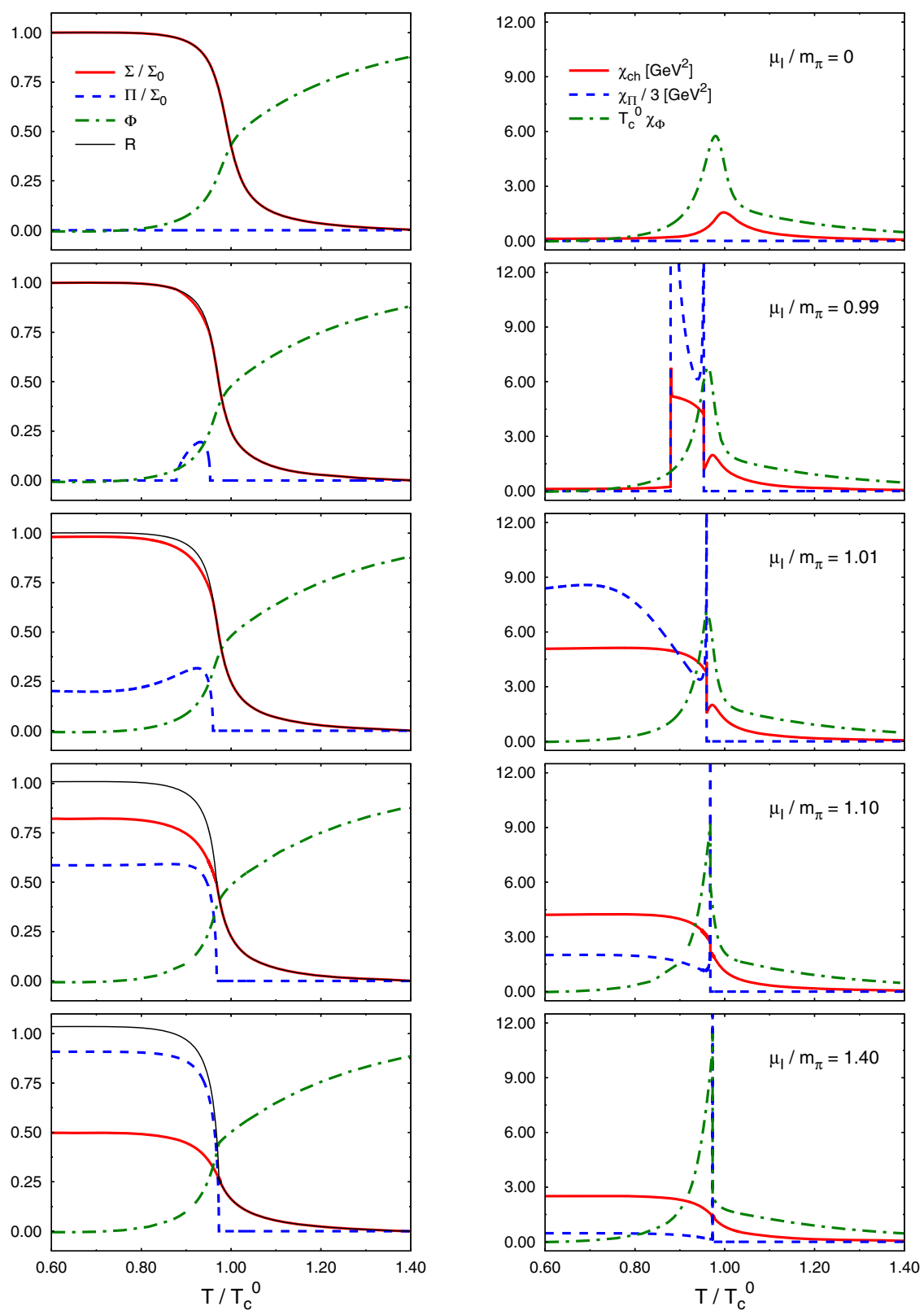

FIG. 6. (Color online) Left: numerical results for the normalized $\Sigma$ and $\Pi$ condensates, the traced Polyakov loop $\Phi$ and the quantity $R$ as functions of the temperature, for some fixed values of $\mu_{I} / m_{\pi}$. Right: numerical results for the susceptibilities associated to the chiral and pion condensates and the Polyakov loop, as functions of $T / T_{c}^{0}$.

that the curves for $T \lesssim T_{c}^{0}$ are quite different from the ones obtained in the framework of the local PNJL model, for which the discontinuity is found to occur at significantly larger values of $\mu_{I} / m_{\pi}$ (see Fig. 3 of Ref. [65]).

Through the analysis of the quantities in Fig. 6 one can sketch the phase diagram in the $\mu_{I}-T$ plane. This is shown in Fig. 8, where the temperature and the isospin chemical potential are normalized to $T_{c}^{0}$ and $m_{\pi}$, respectively. As expected, for low values of $T$ and $\mu_{I}$ the system lies in a "normal matter" (NM) phase, i.e., a $\mathrm{U}(1)_{I_{3} A}$ symmetry broken phase in which the scalar quark-antiquark condensate $\Sigma$ is large and the pion condensate $\Pi$ is zero. By increasing the temperature one reaches a transition to a "quark gluon plasma" (QGP) phase, in which quarks deconfine and the chiral symmetry becomes partially restored. Both chiral restoration and deconfinement transitions occur as smooth crossovers, at approximately a common temperature that does not depend significantly on $\mu_{I}$. The corresponding curves, obtained from the peaks of $\chi_{\mathrm{ch}}$ and $\chi_{\Phi}$ susceptibilities, are shown by the solid and dash-dotted lines in the figure, respectively. The results are found to be similar to those obtained from lattice QCD calculations in Ref. [25], shown by the gray and blue bands. On the other hand, for temperatures below the critical value $T_{c}^{0}$, by increasing the 

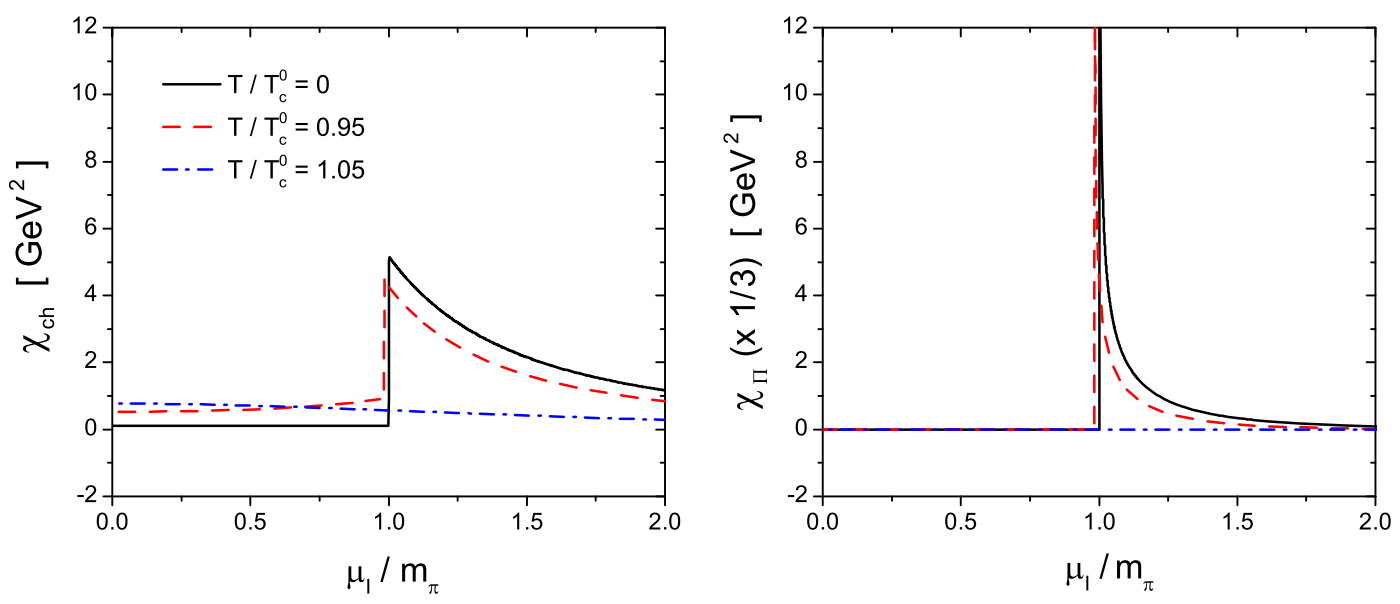

FIG. 7. (Color online) Chiral (left) and pion (right) condensate susceptibilities as functions of the isospin chemical potential, for some representative values of the temperature.

isospin chemical potential one reaches a second-order phase transition to a pion-condensate $(\pi \mathrm{C})$ region in which the condensate $\Pi$ is nonvanishing and therefore the $\mathrm{U}(1)_{I_{3}}$ symmetry is broken. The onset of this phase, shown by the dashed line in Fig. 8, occurs approximately at $\mu_{I}=m_{\pi}$ for all temperature values up to $T_{c}^{0}$, in agreement with lattice QCD calculations (red band in the figure) [25]. Then, for $\mu_{I}>m_{\pi}$, at a given critical temperature there is a secondorder phase transition from the $\pi \mathrm{C}$ phase to the QGP phase. As discussed above, this critical temperature is slightly lower than $T_{c}^{0}$ and remains approximately constant

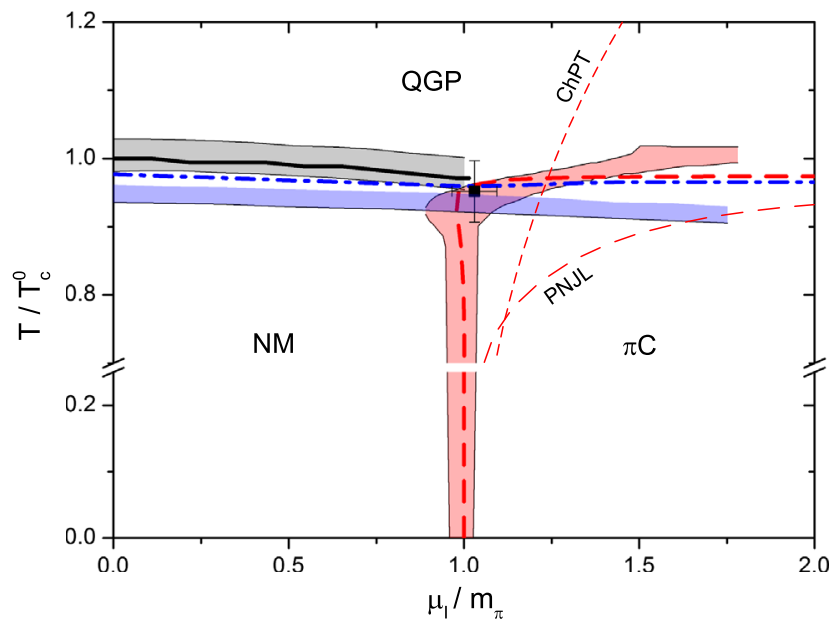

FIG. 8. (Color online) Phase diagram in the $\mu_{I}-T$ plane for the nonlocal PNJL model. NM, QGP and $\pi \mathrm{C}$ stand for normal matter, quark gluon plasma and pion condensation phases, respectively. Solid (black), dashed (red) and dash-dotted (blue) lines correspond to chiral restoration, pion condensation and deconfinement transitions, while the shaded bands indicate the transition regions obtained from LQCD results in Ref. [25]. The thin dashed and short-dashed lines indicate the NM- $\pi \mathrm{C}$ transition curves arising from the local PNJL model and leading-order ChPT, respectively. for all considered values of $\mu_{I}$ above the pion mass. The location of the pseudotriple point where NM, QGP, and $\pi \mathrm{C}$ phases meet is found to be in good agreement with the result obtained in lattice QCD calculations, given by the black square. Concerning the $\mathrm{U}(1)_{I_{3} A}$ symmetry within the $\pi \mathrm{C}$ phase, it is seen that for a given temperature $T$ lower than $T_{c}^{0}$ the values of the quark condensates decrease steadily if $\mu_{I}$ gets increased beyond $m_{\pi}$. This can be read from the values of $\Sigma / \Sigma_{0}$ shown in the left panels of Fig. 6. Notice that for $\mu_{I} \simeq$ $1.4 m_{\pi}$ the value of $\Sigma$ is found to be reduced to approximately one half of the $\mu_{I}=0$ value $\Sigma_{0}$. In addition, in Fig. 8 we show for comparison the $\pi \mathrm{C}-\mathrm{NM}$ transition curves corresponding to the local PNJL model and leading-order chiral perturbation theory [50] (thin dashed and short-dashed lines, respectively). As anticipated in the discussion concerning Fig. 7, it is seen that there is a substantial difference between nonlocal and local PNJL-like approaches. This situation does not change significantly if one considers the NJL model omitting the interaction with the Polyakov loop.

Finally, we have considered the case of a logarithmic PL potential (as proposed e.g., in Ref. [40]), analyzing its effects on the $\mu_{I}-T$ phase diagram. It is worth noticing that, in general, the logarithmic potential leads to a sharper chiral restoration transition [35]. Consequently, for parametrizations leading to a quark condensate $\left|\Sigma_{0}\right| \lesssim(240 \mathrm{MeV})^{3}$ this transition is found to be of first order at $\mu_{I}=0$ for $T_{0}=210 \mathrm{MeV}$. Taking a parametrization for which $\Sigma_{0}=-(250 \mathrm{MeV})^{3}$, it is seen that the agreement with LQCD results-in particular, for the case of the NM- $\pi$ C transition line-is similar to the one shown in Fig. 8 for the polynomial PL potential.

\section{CONCLUSIONS}

We have analyzed the phase diagram of strongly interacting matter within a nonlocal two-flavor PNJL model, 
considering both zero and finite temperature and nonzero isospin chemical potential. In this context, we have studied the quark deconfinement and the breakdown/restoration of chiral and isospin symmetries, together with the corresponding footprints on various thermodynamic quantities.

At zero temperature, for $\mu_{I}=m_{\pi}$ one finds the onset of a phase in which isospin symmetry is broken by the presence of a nonzero pion condensate. Up to $\mu_{I} \simeq 2 m_{\pi}$, one observes a rapid growth of this condensate, in overall agreement with the predictions from other effective model analyses and LQCD calculations. The agreement is also good for various thermodynamic quantities, as the pressure, energy density, isospin particle density, and interaction energy. For larger values of $\mu_{I}$ (where no LQCD data are available up to now), although one finds some general agreement in the qualitative behavior of these quantities, there are significant quantitative discrepancies between the results from different theoretical approaches.

In the case of a system at finite temperature, for low values of $\mu_{I}$ the pion condensate is absent and one gets, as expected, a transition from the usual "normal matter" scenario into a quark-gluon plasma phase in which chiral symmetry is restored and quarks are deconfined. This transition proceeds as a smooth crossover signaled by the behavior of chiral and Polyakov loop susceptibilities. The critical temperature $T_{c}^{0} \simeq 174 \mathrm{MeV}$ is approximately the same for both chiral restoration and quark deconfinement. For $T \leq T_{c}^{0}$, by increasing the isospin chemical potential one finds a second-order transition into a pion condensation $(\pi \mathrm{C})$ phase, in which isospin symmetry is spontaneously broken. The corresponding critical line $\mu_{I}(T)$ is a primary result of our analysis. The critical value
$\mu_{I}=m_{\pi}$ found at $T=0$ remains approximately constant up to $T \simeq T_{c}^{0}$, reaching a pseudotriple point in which NM, QGP, and $\pi \mathrm{C}$ phases coexist. It can be seen that there is a remarkable agreement between these results and those obtained from lattice QCD calculations. On the other hand, the $\pi \mathrm{C}$-QGP transition occurs at a temperature of the order of $T_{c}^{0}$, which is approximately constant for $\mu_{I}>m_{\pi}$. It is worth noticing that our predictions for the border of the $\pi \mathrm{C}$ phase region (and, in particular, for the location of the triple point) are in good agreement with the available results from lattice QCD, whereas they differ significantly from the predictions obtained within chiral perturbation theory and from those found in the framework of the local PNJL model.

As a natural extension of this work, it would be worth studying the phase diagram for the case of both nonzero isospin and baryonic chemical potentials. In addition, it would be interesting to consider in this context the existence of pion stars, a potential new class of compact objects formed by a Bose-Einstein condensate of charged pions and a gas of leptons. We expect to report on these issues in the near future.

\section{ACKNOWLEDGMENTS}

This work has been supported in part by Consejo Nacional de Investigaciones Científicas y Técnicas and Agencia Nacional de Promoción Científica y Tecnológica (Argentina), under Grants No. PIP17-700 and No. PICT1703-0571, respectively, and by the National University of La Plata (Argentina), Project No. X824.
[1] K. Fukushima and T. Hatsuda, Rep. Prog. Phys. 74, 014001 (2011).

[2] M. G. Alford, A. Schmitt, K. Rajagopal, and T. Schäfer, Rev. Mod. Phys. 80, 1455 (2008).

[3] L. McLerran and R. D. Pisarski, Nucl. Phys. A796, 83 (2007).

[4] K. Fukushima, Phys. Rev. D 77, 114028 (2008); 78, 039902 (E) (2008).

[5] K. Fukushima, T. Kojo, and W. Weise, Phys. Rev. D 102, 096017 (2020).

[6] K. Fukushima and T. Kojo, Astrophys. J. 817, 180 (2016).

[7] L. McLerran and S. Reddy, Phys. Rev. Lett. 122, 122701 (2019).

[8] T. Schäfer and F. Wilczek, Phys. Rev. Lett. 82, 3956 (1999).

[9] M. G. Alford, J. Berges, and K. Rajagopal, Nucl. Phys. B558, 219 (1999).

[10] T. Hatsuda, M. Tachibana, N. Yamamoto, and G. Baym, Phys. Rev. Lett. 97, 122001 (2006).

[11] E. Nakano and T. Tatsumi, Phys. Rev. D 71, 114006 (2005).
[12] M. Buballa and S. Carignano, Prog. Part. Nucl. Phys. 81, 39 (2015).

[13] D. Nickel, Phys. Rev. D 80, 074025 (2009).

[14] J. P. Carlomagno, D. Gómez Dumm, and N. N. Scoccola, Phys. Rev. D 92, 056007 (2015).

[15] M. G. Alford, A. Kapustin, and F. Wilczek, Phys. Rev. D 59, 054502 (1999).

[16] A. Cherman, M. Hanada, and D. Robles-Llana, Phys. Rev. Lett. 106, 091603 (2011).

[17] A. Cherman and B. C. Tiburzi, J. High Energy Phys. 06 (2011) 034.

[18] M. Hanada and N. Yamamoto, J. High Energy Phys. 02 (2012) 138.

[19] J. B. Kogut and D. K. Sinclair, Phys. Rev. D 66, 014508 (2002).

[20] J. B. Kogut and D. K. Sinclair, Phys. Rev. D 66, 034505 (2002).

[21] J. B. Kogut and D. K. Sinclair, Phys. Rev. D 70, 094501 (2004). 
[22] P. de Forcrand, M. A. Stephanov, and U. Wenger, Proc. Sci. LATTICE2007 (2007) 237 [arXiv:0711.0023].

[23] P. Cea, L. Cosmai, M. D’Elia, A. Papa, and F. Sanfilippo, Phys. Rev. D 85, 094512 (2012).

[24] W. Detmold, K. Orginos, and Z. Shi, Phys. Rev. D 86, 054507 (2012).

[25] B. B. Brandt, G. Endrodi, and S. Schmalzbauer, Phys. Rev. D 97, 054514 (2018).

[26] B. B. Brandt, G. Endrodi, E. S. Fraga, M. Hippert, J. Schaffner-Bielich, and S. Schmalzbauer, Phys. Rev. D 98, 094510 (2018).

[27] D. T. Son and M. A. Stephanov, Phys. Rev. Lett. 86, 592 (2001).

[28] M. Mannarelli, Particles 2, 411 (2019).

[29] D. Blaschke, M. Buballa, A. E. Radzhabov, and M. K. Volkov, Yad. Fiz. 71, 2012 (2008).

[30] G. A. Contrera, D. Gomez Dumm, and N. N. Scoccola, Phys. Lett. B 661, 113 (2008).

[31] G. A. Contrera, D. Gomez Dumm, and N. N. Scoccola, Phys. Rev. D 81, 054005 (2010).

[32] G. A. Contrera, M. Orsaria, and N. N. Scoccola, Phys. Rev. D 82, 054026 (2010).

[33] T. Hell, S. Roessner, M. Cristoforetti, and W. Weise, Phys. Rev. D 79, 014022 (2009).

[34] T. Hell, S. Rossner, M. Cristoforetti, and W. Weise, Phys. Rev. D 81, 074034 (2010).

[35] D. Gomez Dumm, J. P. Carlomagno, and N. N. Scoccola, Symmetry 13, 121 (2021).

[36] P. N. Meisinger and M. C. Ogilvie, Phys. Lett. B 379, 163 (1996).

[37] K. Fukushima, Phys. Lett. B 591, 277 (2004).

[38] E. Megias, E. Ruiz Arriola, and L. L. Salcedo, Phys. Rev. D 74, 065005 (2006).

[39] C. Ratti, M. A. Thaler, and W. Weise, Phys. Rev. D 73, 014019 (2006).

[40] S. Roessner, C. Ratti, and W. Weise, Phys. Rev. D 75, 034007 (2007).

[41] S. Mukherjee, M. G. Mustafa, and R. Ray, Phys. Rev. D 75, 094015 (2007).

[42] C. Sasaki, B. Friman, and K. Redlich, Phys. Rev. D 75, 074013 (2007).

[43] S. Noguera and N. N. Scoccola, Phys. Rev. D 78, 114002 (2008).

[44] E. Ruiz Arriola and L. L. Salcedo, Phys. Lett. B 450, 225 (1999).

[45] D. Blaschke, Y. L. Kalinovsky, G. Roepke, S. M. Schmidt, and M. K. Volkov, Phys. Rev. C 53, 2394 (1996).

[46] L. y. He, M. Jin, and P. f. Zhuang, Phys. Rev. D 71, 116001 (2005).
[47] S. S. Avancini, A. Bandyopadhyay, D. C. Duarte, and R. L. S. Farias, Phys. Rev. D 100, 116002 (2019).

[48] Z. Zhang and Y. X. Liu, Phys. Rev. C 75, 064910 (2007).

[49] T. Sasaki, Y. Sakai, H. Kouno, and M. Yahiro, Phys. Rev. D 82, 116004 (2010).

[50] K. Splittorff, D. Toublan, and J. J. M. Verbaarschot, Nucl. Phys. B639, 524 (2002).

[51] P. Adhikari, J. O. Andersen, and P. Kneschke, Eur. Phys. J. C 79, 874 (2019).

[52] P. Adhikari, J. O. Andersen, and M. A. Mojahed, Eur. Phys. J. C 81, 173 (2021).

[53] D. Gomez Dumm, A. G. Grunfeld, and N. N. Scoccola, Phys. Rev. D 74, 054026 (2006).

[54] D. Gomez Dumm, S. Noguera, and N. N. Scoccola, Phys. Lett. B 698, 236 (2011).

[55] G. Ripka, Quarks Bound By Chiral Fields: The QuarkStructure of the Vacuum and of Light Mesons and Baryons (Oxford University Press, Oxford, 1997).

[56] B. J. Schaefer and J. Wambach, Phys. Rev. D 75, 085015 (2007).

[57] V. Skokov, B. Stokic, B. Friman, and K. Redlich, Phys. Rev. C 82, 015206 (2010).

[58] T. K. Herbst, J. M. Pawlowski, and B. J. Schaefer, Phys. Rev. D 88, 014007 (2013).

[59] A. E. Radzhabov, D. Blaschke, M. Buballa, and M. K. Volkov, Phys. Rev. D 83, 116004 (2011).

[60] D. Ebert, K. G. Klimenko, V. C. Zhukovsky, and A. M. Fedotov, Eur. Phys. J. C 49, 709 (2007).

[61] O. Scavenius, A. Dumitru, and J. T. Lenaghan, Phys. Rev. C 66, 034903 (2002).

[62] B. J. Schaefer, J. M. Pawlowski, and J. Wambach, Phys. Rev. D 76, 074023 (2007).

[63] B. J. Schaefer, M. Wagner, and J. Wambach, Phys. Rev. D 81, 074013 (2010).

[64] D. Gomez Dumm and N. N. Scoccola, Phys. Rev. C 72, 014909 (2005).

[65] Z. Y. Lu, C. J. Xia, and M. Ruggieri, Eur. Phys. J. C 80, 46 (2020).

[66] B. B. Brandt and G. Endrodi, Proc. Sci. LATTICE2016 (2016) 039 [arXiv:1611.06758].

[67] B. B. Brandt, G. Endrodi, and S. Schmalzbauer, EPJ Web Conf. 175, 07020 (2018).

[68] B. B. Brandt, G. Endrodi, and S. Schmalzbauer, Proc. Sci. Confinement2018 (2018) 260 [arXiv:1811.06004].

[69] J. P. Carlomagno, Phys. Rev. D 97, 094012 (2018).

[70] J. P. Carlomagno, D. Gómez Dumm, and N. N. Scoccola, Phys. Rev. D 88, 074034 (2013).

[71] J. B. Kogut and D. Toublan, Phys. Rev. D 64, 034007 (2001).

[72] S. Carignano, A. Mammarella, and M. Mannarelli, Phys. Rev. D 93, 051503 (2016). 